\title{
Microbiological Profile of Cases of Community Acquired Pneumonia and Antimicrobial Susceptibility Testing of the Etiological Agents with Special Reference to Streptococcus pneumoniae
}

\author{
Jasmine Vinshia ${ }^{*}$, C. Suja ${ }^{1}$ and P.K. Uma Maheshwari ${ }^{2}$ \\ ${ }^{1}$ Department of Microbiology, Rajas Dental College \& Hospital, Tirunelveli, India \\ ${ }^{2}$ Departmentof Microbiology, SRM Medical College Hospital \& Research Centre, \\ Chennai, India \\ *Corresponding author
}

\begin{abstract}
Keywords
Community

Acquired

Pneumonia,

Streptococcus

pneumoniae,

Oxacillin,

Levofloxacin

Article Info

Accepted:

15 January 2019

Available Online:

10 February 2019 identify microbial etiology of cases of Community acquired pneumonia, perform antibiotic susceptibility of the isolates \& compare the anti-microbial resistance pattern with special reference to Streptococcus pneumoniae. The study was carried out at SRM Medical College Hospital \& Research Centre from February 2012 to February 2013. A total of 619 cases with Community acquired pneumonia were included in the study. Sputum from the clinically proven subjects was collected and processed using standard microbiological techniques. Among the 619 clinical samples, a predominant number $(n=224)$ grew Streptococcus pneumoniae which accounts for $37 \%$ of the total isolates followed by Klebsiella pneumoniae $(7 \%, \mathrm{n}=44)$, Moraxella $(4 \%, \mathrm{n}=29)$, Pseudomonas $(4 \%, \mathrm{n}=25)$, Acinetobacter $(2 \%, \mathrm{n}=13)$, Staphylococcus aureus $(2 \%, \mathrm{n}=12)$ and Non-fermenting Gram negative bacilli $(1 \%, \mathrm{n}=6)$ respectively. $40 \%(\mathrm{n}=247)$ of the samples grew normal flora while $3 \%(n=19)$ of the samples were considered insignificant since sputum Gram stain findings and culture results were discordant. The causative agent, Streptococcus pneumoniae historically was susceptible to penicillin and many other antimicrobial agents. With the background of changing trends in the etiology of Community acquired pneumonia and also with reference to the antimicrobial therapy this study proves that along with penicillin, resistance has emerged to other agents including Cotrimoxazole and macrolides.
\end{abstract}

A B S T R A C T

Community acquired pneumonia is an infection of pulmonary parenchyma. Despite of advancements in antimicrobial therapy, it stills remains a major threat with significant mortality and morbidity. It accounts for about $13-18 \%$ of all nosocomial infections. To

\section{Introduction}

Pneumonia refers to inflammation of the distal lung caused by infection with microorganisms and is characterized histologically by the accumulation of neutrophils in the distal bronchioles, alveoli and interstitium (Langer et al., 1987). It is the second most common form of nosocomial infection that accounts for $13 \%$ to $19 \%$ of all infections. 
The burden of pneumonia is a significant and increasing problem associated with the present day healthcare system.

Pneumonia can be Community-acquired pneumonia and Nosocomial pneumonia.

Community acquired pneumonia is defined as pulmonary infiltration of the lung revealed by radiographic examination at the time of admission, including at least two of the following

Fever (Temp > 37.8C)

Production of purulent sputum.

Cough

Leucocytosis (WBC count >10000/cumm) (Archana Choure Chintaman et al., 2017).

The infection is acquired when the causative organism overcomes the primary protective barriers of the body and initiates the infection in the alveoli of the lungs.

CAP is diagnosed by physical examination, $\mathrm{X}$-ray and laboratory investigations. Invasive methods are the most effective methods for diagnosis of CAP but it has drawback of technical difficulty and sample contamination due to oropharyngeal secretions (Bansal et al., 2004; Peto et al., 2014).

The present study was planned to determine changing trends in the etiology of pneumonia with reference to the antimicrobial therapy.

\section{Objectives}

To identify and isolate the microbial etiology of cases of Community acquired Pneumonia.

To perform Antibiotic Susceptibility Testing of the clinically significant isolates and to compare their anti-microbial resistance pattern. To determine the Resistance pattern of Streptococcus pneumoniae with common sulphonamides and macrolides groups of antimicrobial agents.

\section{Materials and Methods}

Period of study: Feb 2012 - Feb 2013

Samples Collected: Appropriately collected Sputum samples from clinically proven CAP cases.

Methodology Used: Processing of specimens

Gram staining.

Culture onto Blood Agar/Chocolate Agar/MacConkey Agar

Identification of the organism to species level Streptococcus pneumoniae using bile solubility and optochin sensitivity test (Fig. 1) Performance of AST for all clinically significant isolates and their comparison as per CLSI guidelines.

Screening for susceptibility pattern for Streptococcus pneumoniae:

Disk-agar diffusion test for penicillin resistance: The adjusted inoculums prepared in THB $\left(10^{8} \mathrm{CFU} / \mathrm{ml}\right)$ was spread onto the dried surface of neo-peptone agar supplemented with5\% defibrinated rabbit blood as described for the Kirby-Bauer method. A 10-U penicillin disk was applied, and the plate was incubated overnight at $37^{\circ} \mathrm{C}$ in a candle jar. The zone of inhibition was measured from the top of the plate with a ruler.

Inoculum preparation: The inocula were prepared from Pneumococci grown overnight on neo-peptone agar supplemented with 5\% defibrinated rabbit blood at $37^{\circ}$. Some of the overnight growth was suspended in MuellerHinton broth (MHB) and adjusted to a turbidity equivalent to a 0.5 McFarland standard (approximately $10^{8}$ colony forming units $[\mathrm{CFU}] / \mathrm{ml})$. For the disk-agar diffusion screening test for resistance to penicillin, the pneumococci were grown overnight in Todd- 
Hewitt broth (THB) at $37^{\circ}$ in a candle jar and diluted with THB to a turbidity equivalent to a 0.5 McFarland standard (Robert Cooksey et $a l)$.

\section{Results and Discussion}

During the study period from February 2012 till February 2013 a total of 619 Community acquired pneumonia cases that were not hospitalized and were treated on an outpatient basis were selected and subjected to microbiological evaluation.

Streptococcus pneumoniae was the predominant organism $(36 \%, \mathrm{n}=224)$ isolated followed by Klebsiella pneumoniae $(7 \%, \mathrm{n}=$ 44), Moraxella (4\%, $\mathrm{n}=29)$, Pseudomonas (4\%, $\mathrm{n}=25)$, Acinetobacter $(2 \%, \mathrm{n}=13)$, Staphylococcus aureus $(2 \%, \mathrm{n}=12)$ and Nonfermenting Gram negative bacilli $(1 \%, \mathrm{n}=6)$ respectively. $40 \% \quad(n=247)$ of the samples grew normal flora while $3 \% \quad(n=19)$ of the samples were considered insignificant since sputum Gram stain findings and culture results were discordant (Graph 1).

Summary of antibiotic resistance profile of predominant isolates of cap (Graph 2)

All isolates of Streptococcus pneumoniae were found to be sensitive to Penicillin.

Resistance to Oxacillin was $56 \%$ by disc diffusion. Resistance to Erythromycin and Cotrimoxazole were $14 \%$ and $44 \%$ respectively.

Isolates of Klebsiella pneumoniae were $95 \%$ resistant to Cefotaxime, $50 \%$ to Ceftazidime, $30 \%$ to Cefopodoxime and $43 \%$ to Meropenem.

$35 \%$ and $8 \%$ Resistance to Ciprofloxacin and Amoxyclav by Moraxella spp were observed.
Drug resistance profile of Streptococcus pneumoniae

From a total of 619 isolates, 224 were identified as Streptococcus pneumoniae which accounts for about $36 \%$ of the total number of organisms isolated. The Antibiotic resistance pattern of the isolated Streptococcus strains were determined using Standard Macrolides, fluoroquinolones, sulphonamides, penicillin and $\beta$ - lactamase class of antibiotics by disc diffusion KirbyBauer method (Fig. 2).

The resistance pattern was found to be penicillin (oxacillin) 40\%, Cotrimoxazole (25\%), erythromycin (35\%), Amoxyclav $(\mathrm{n}=26,12 \%)$ and Levofloxacin $(\mathrm{n}=12,5 \%)$ respectively (Graph 3).

MIC determination by E-test for Strip for penicillin didn't yield consistent result. Whereas E-test Strip gave validated results for Levofloxacin (Fig. 3). The differences in study patterns may be likely due to varying study designs, dates the studies were performed, laboratory techniques, population groups and local patterns of antimicrobial use (Stephen M. Ostroff et al., 1996). This study carried out in SRM MCH \& RC, observed the microbial etiology of cases of Community acquired pneumonia that were not hospitalised but were treated in an out-patient basis. During the study period from February 2012 till February 2013, a total of 619 cases of Community acquired pneumonia were selected and subjected to microbiological evaluation. The isolation rates of organisms from cases of community acquired pneumonia were $37 \%$ of Streptococcus pneumoniae being the predominant organism followed by Klebsiella pneumoniae (7\%), Moraxella (4\%), Pseudomonas (4\%), Acinetobacter (2\%) and Staphylococcus aureus (2\%) respectively. 
Int.J.Curr.Microbiol.App.Sci (2019) 8(2): 1989-1997

Fig.1 Optochin sensitivity on blood agar

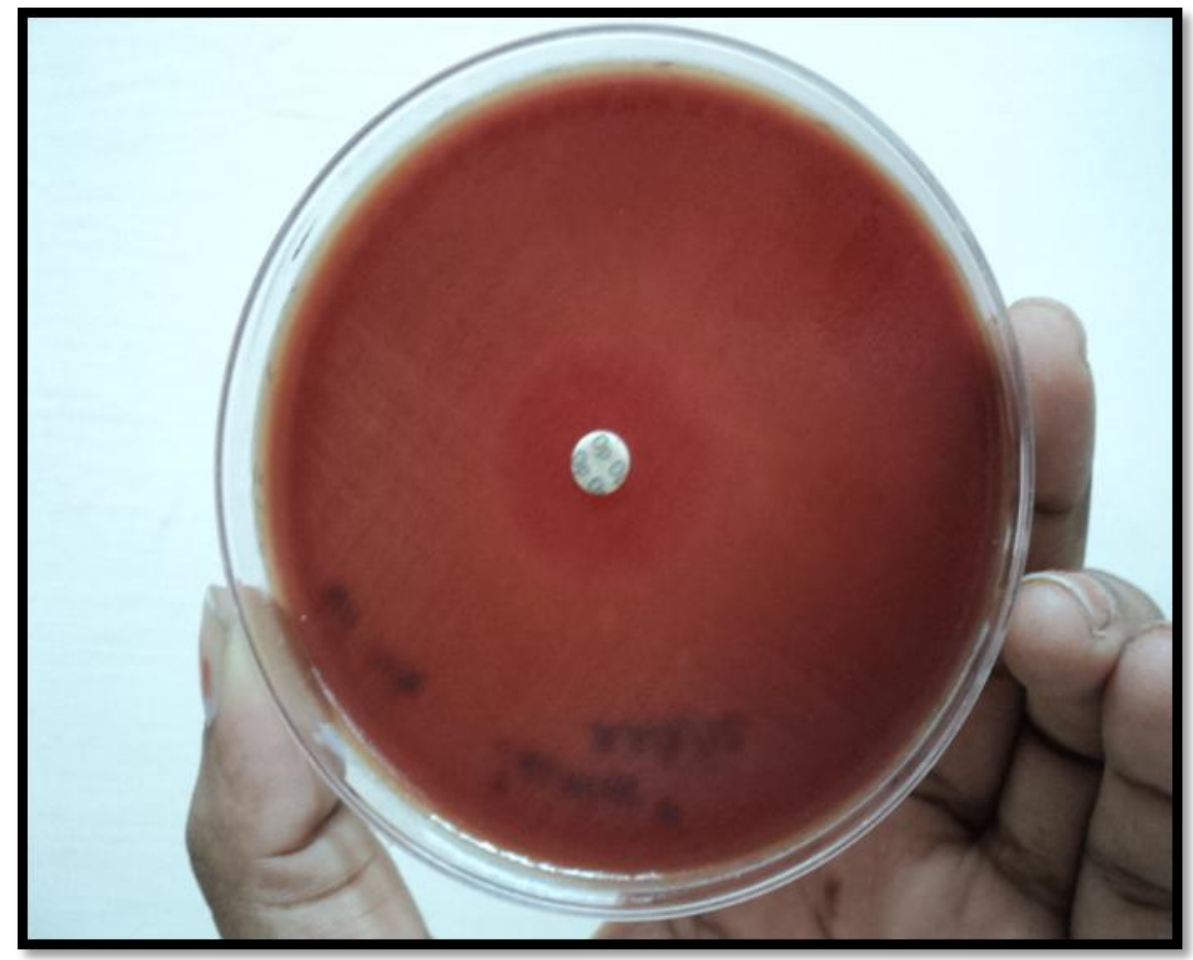

Fig.2 AST pattern of Streptococcus pneumoniae

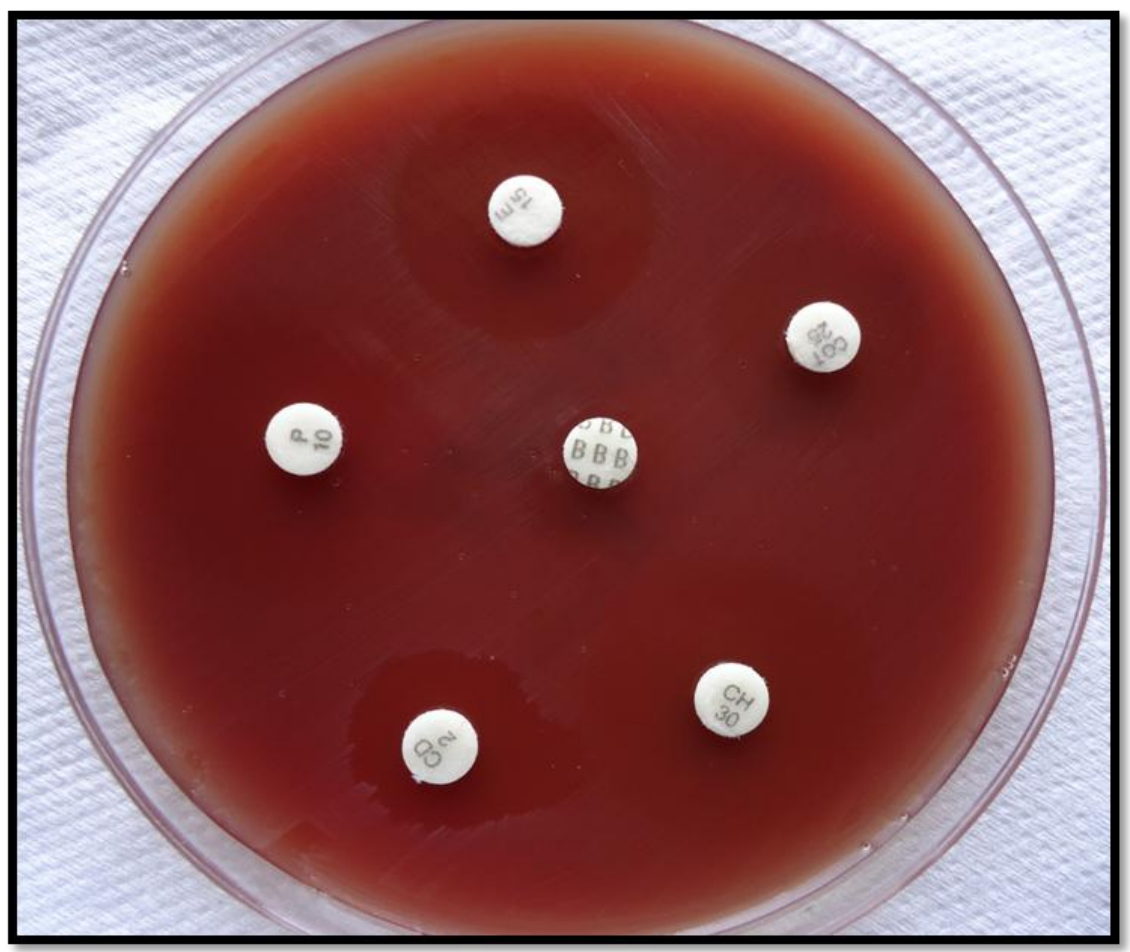


Fig.3a MIC by E-test-Levofloxacin susceptible isolate (MIC $2 \mu \mathrm{g} / \mathrm{ml}$ )

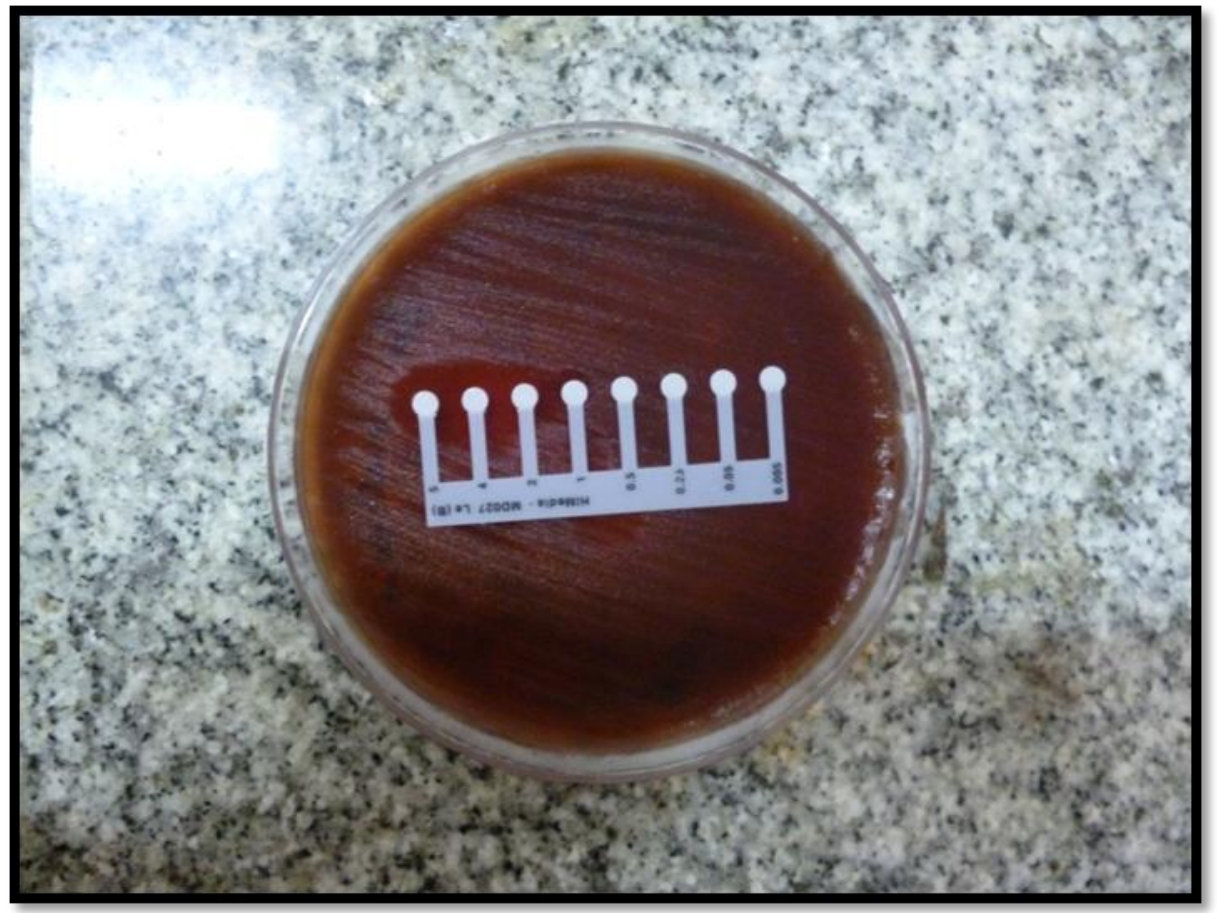

Fig.3b MIC by E-test- Levofloxacin resistant isolate (MIC $>2 \mu \mathrm{g} / \mathrm{ml}$ )

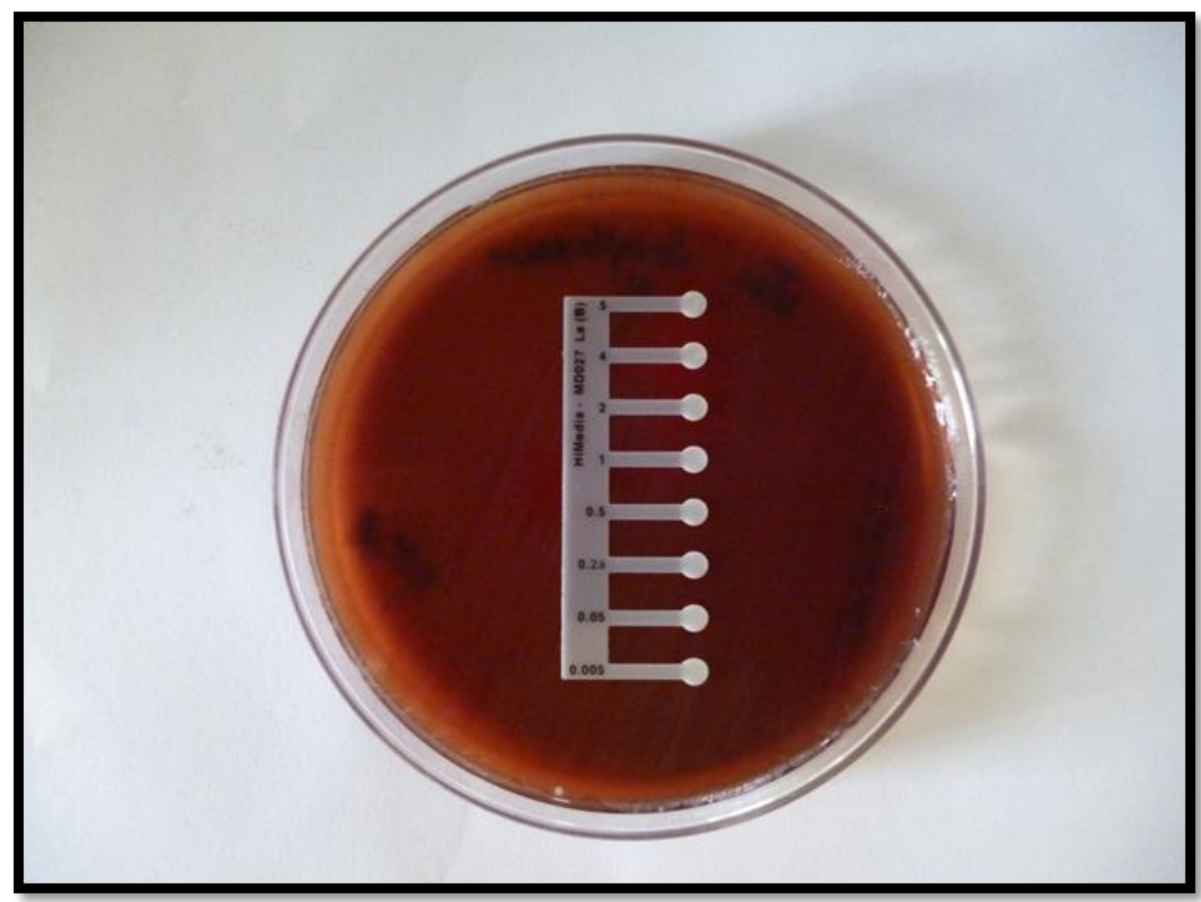




\section{Graph.1}

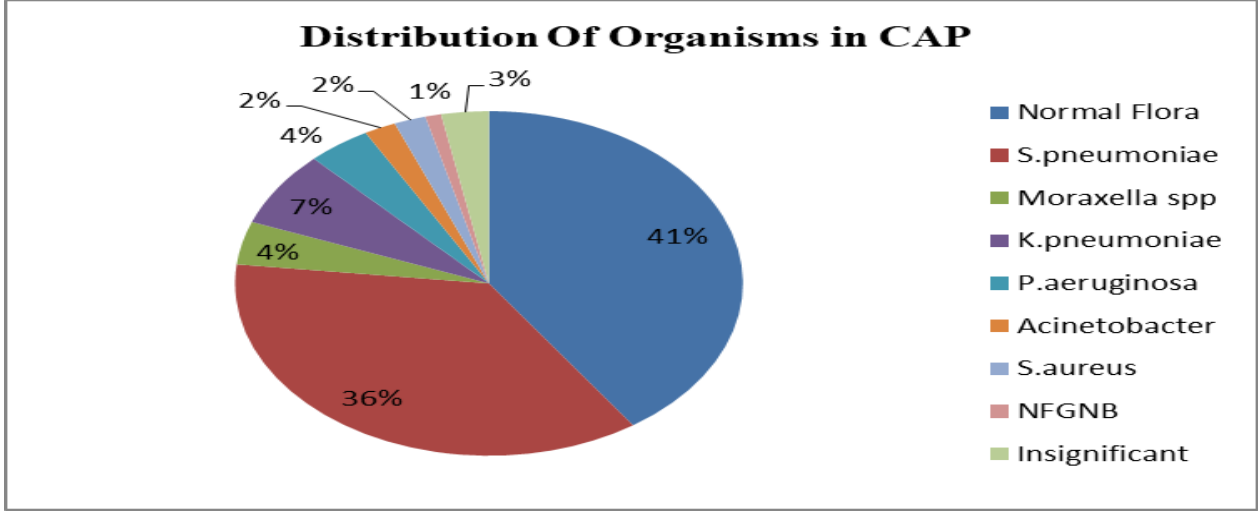

Graph.2 Resistance profile of all isolates from cases of CAP

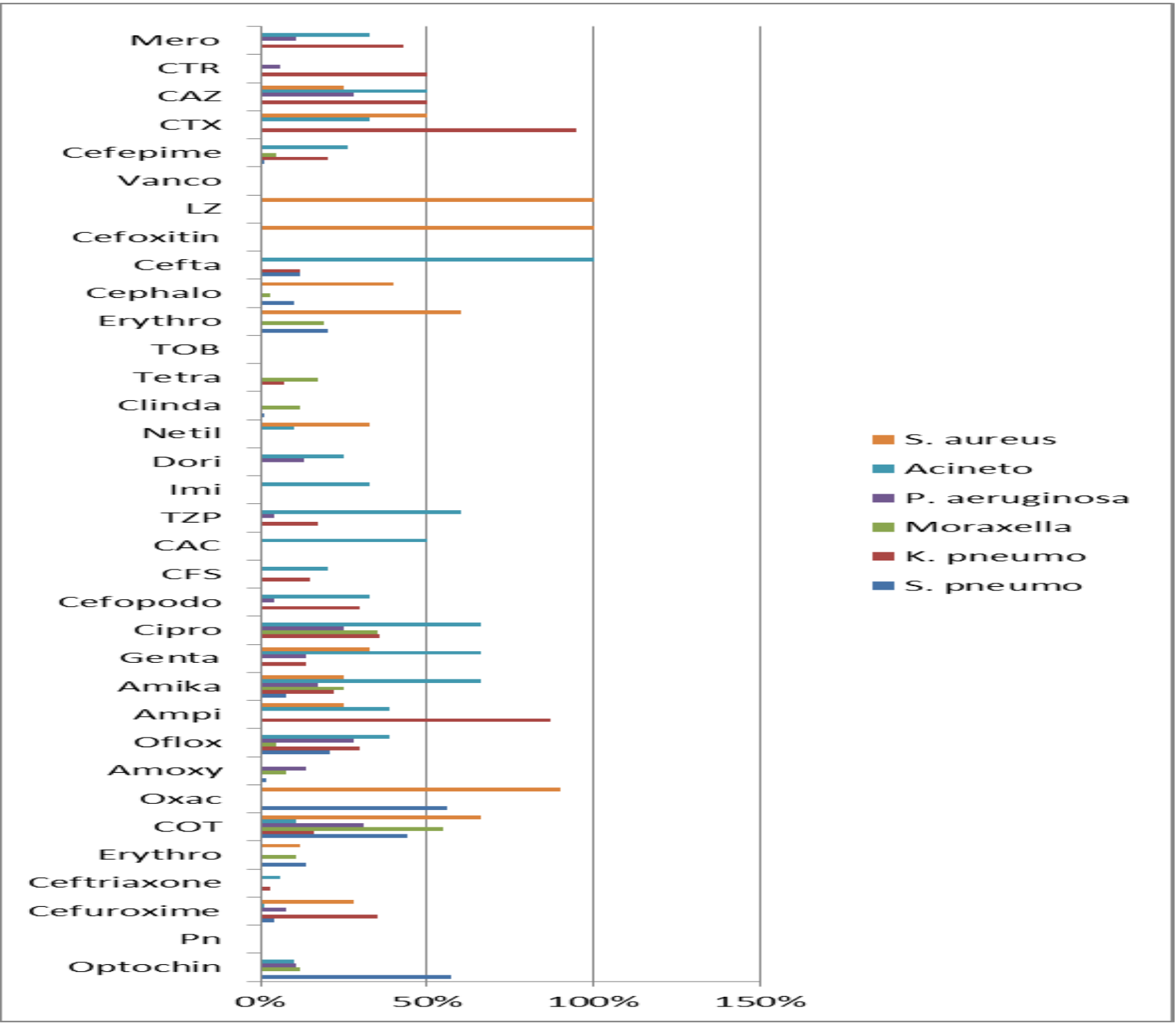




\section{Graph.3}

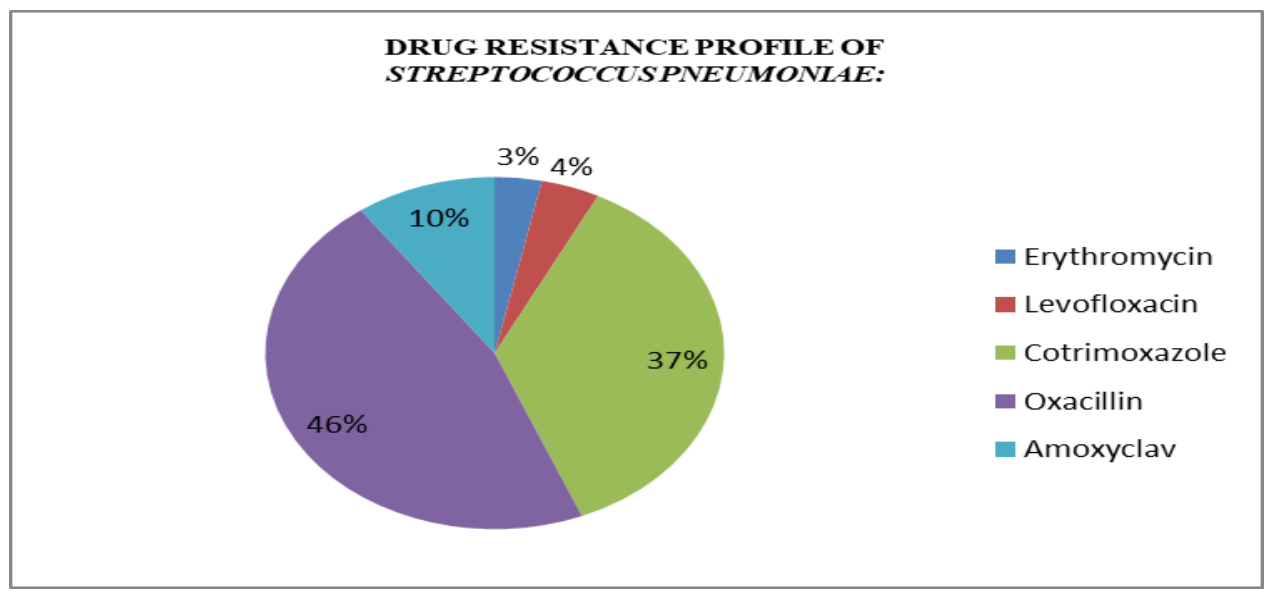

$3 \%$ of the samples were considered insignificant since sputum Gram stain findings and culture results were discordant.

The total number of isolates of Streptococcus pneumoniae was 224 which accounts for $36 \%$. All these were isolates from sputum samples obtained from patients with pneumonia.

No invasive pneumococcal isolates were included in the study.

Streptococcus pneumoniae historically was susceptible to penicillin and many other antimicrobial agents. This is no longer true. (Butler et al).

Data from literature indicates that in some countries as many as $40 \%$ of strains are intermediate or resistant to penicillin (Swen Son et al., 1986).

Along with penicillin, resistance has emerged to other agents, including cephalosporins, macrolides and co-trimoxazole (Bradley et al., 1997).

It is now essential that laboratories test strains of Streptococcus pneumoniae for resistance to these agents.
In a study conducted in Greece, disc diffusion tests revealed $14 \%$ of sputum isolates were resistant to penicillin (Kanavaki et al., 1994).

The resistance rates of pneumococcal isolates from various body sites to various drugs such as penicillin resistant (19\%) and cotrimoxazole (43\%) (Shibl et al., 1992).

Our study correlates with the prospective study done in Bombay to access the incidence of Hospital-acquired pneumonia, One hundred and sixty-eight patients developed nosocomial pneumonia. Common isolates included Pseudomonas spp (44\%) and Klebsiella spp (34\%). The most frequently used antibiotics were cefotaxime (34\%), amikacin (25\%), gentamicin (23\%) and ofloxacin (13\%).

In conclusion,

Microbial etiology of CAP shows wide variety of causative organisms.

Streptococcus pneumoniae is the predominant organism in CAP.

Appropriate sputum collection, transport, processing and validation are essential in arriving at microbiological diagnosis. Utility of Gram stain is two fold. First, provides the idea regarding the choice of empirical 
antibiotic. Second, the yield of the culture is to be consistent with the Gram stain finding for interpretation. Yield of no growth in sputum culture should not be considered negative. It rules out rare organisms.

Organisms such as Hemophilus influenzae, Legionella were not looked for in the present study.

Risk factors that are modifiable has to be put into practice as per CDC recommendations and guidelines for prevention of HCAP.

ICU settings warrants surveillance and infection control measures and periodic surveillance to assess the trends in susceptibility patterns are essential to decrease the emergence of resistant organisms both in community and hospital settings.

Role of Carbapenems and Resistance to various agents in the same class varies. Identification of etiological agent in CAP is very important to start the appropriate antimicrobial drug. It is essential because indiscriminate use of antibiotics had led to wide spread emergence of multidrug resistant pathogens.

The profile of bacterial agents varies with the geographical area, so it is necessary to do the surveillance to find out the exact causative agents. This will help to form the proper antibiotic policy for that particular hospital, which in turn will reduce the patient mortality and morbidity.

\section{Acknowledgement}

I would like to thank The Lord Almighty for his gracious abundance in all paths of my life, also I would like to extend my gratitude to my family, my teachers and my whole department for their guidance and extended help in completion of my research work.

\section{References}

Archana Choure Chintaman, Dnyaneshwari P. Ghadage and Arvind V. Bhore.
Bacteriological Profile of Community Acquired Pneumonia in a Tertiary Care Hospital. Int.J.Curr.Microbiol. App.Sci (2017) 6(4): 190-194.

Bansal, S,. Kashyap, S., Pal, L.S., Goel, A. 2004. Clinical and Bacteriological profile of community acquired pneumonia in Shimla, Himachal Pradesh. Indian J. Chest Dis. Allied Sci., 46: 17-22.

Bradley, J.S., Scheld, W.M., et al., 1997. The challenge of penicillin resistant Streptococcus pneumoniae meningitis: current antibiotic therapy in the 1990s. Clin. Infect. Dis. 24:S213-S221.

Butler, J.C., Hofmann, J., Cetron, M.S., et al., 1996. The continued emergence of drug-resistant Streptococcus pneumoniae in the United States: an update from the Centres for Disease Control and Prevention's pneumococcal surveillance system. J. Clin. Microbiol. 174: 986-993.

Kanavaki S, et al., Antibiotic resistance of clinical isolates of Streptococcus pneumoniae in Greece. $\mathrm{J}$ Clin Microbiol 1994; 32: 3056-8.

Langer M, Cigada M, Mandelli M et al., Early onset pneumonia: a multicenter study in intensive care units. Intensive Care Med 1987; 13: 342-346.

Robert Cooksey, et al, Antimicrobial Susceptibility Patterns of Streptococcus pneumoniae. Antimicrob Agents Chemother. 1978 Apr; 13(4): 645-648.

Shibl AM, et al., Surveillance of Streptococcus pneumoniae serotypes in Riyadh and their susceptibility to penicillin and other commonly prescribed antibiotics. J Antimicrob Chemother., 1992; 29:149-57.

Stephen M Ostroff, Lee H, et al., 1996. Resistance patterns of Streptococcus pneumoniae and Haemophilus 
influenzae isolates recovered in Egypt

penicillin resistance. J. Clin. from children with pneumonia.

Microbiol., 24:749.

Swenson, J.M., Hill, B.C. Thronsberry, C., et

al., 1986. Screening pneumococci for

\section{How to cite this article:}

Jasmine Vinshia, C. Suja and Uma Maheshwari, P.K. 2019. Microbiological Profile of Cases of Community Acquired Pneumonia and Antimicrobial Susceptibility Testing of the Etiological Agents with Special Reference to Streptococcus pneumoniae. Int.J.Curr.Microbiol.App.Sci. 8(02): 1989-1997. doi: https://doi.org/10.20546/ijcmas.2019.802.233 